\title{
The Role of Sitters in Delirium: an Update
}

Frances M. Carr, MBChB

Internal Medicine Department, University of Saskatchewan, Saskatoon, SK

DOI:http://dx.doi.org/10.5770/cgj.16.29

\begin{abstract}
Purpose

The concept behind constant observation is not new. Whilst traditionally performed by nursing staff, it is now commonly performed by sitters. Details surrounding the usage, job description, training, clinical and cost effectiveness of sitters are not known; hence the reason for this review.
\end{abstract}

\section{Methods}

A literature search was performed in MEDLINE, Cochrane Database of Systematic Reviews, and PubMed from the years 1960 to October 2011. The definition for sitter used in the articles was accepted for this review.

\section{Results}

From this review, it is evident that sitters are being employed in a variety of settings. The question of which type of person would provide the most benefit in the sitter role is still not clear; whilst sitters have typically included family and volunteers, it may be trained volunteers who may offer the most cost-effective solution. The paucity of information available regarding the training and assessments of sitters and the lack of formal guidelines regulating sitters' use results in a lack of information available regarding these sitters, and current available evidence is conflicting regarding the benefits in terms of cost and clinical outcome. The only strong evidence relating to clinical benefit comes from the use of fully-trained sitters as part of a multi-interventional program (i.e., HELP)

\section{Conclusions}

Current evidence supports a role for the sitter as part of the management of patients with delirium. The most costeffective sitter role appears to be trained volunteers. Further research is needed to determine the specific type of training required for the sitter role. The creation of a national set of regulations or guidelines would provide safeguards in the industry to ensure safe and effective patient care.
Key words: delirium, constant observation, sitters, volunteers

\section{INTRODUCTION}

Delirium is an extremely common problem occurring in over $50 \%$ of older, hospitalized patients, and is associated with both a decline in functional ability and increased morbidity and mortality. Consequently, it has been referred to as being 'an independent marker of functional decline'.(1) In addition, the development of delirium is associated with higher rates of falling, ${ }^{(2)}$ contributing to poor clinical outcomes. The hospital costs are considerably higher for these patients compared to those without delirium. ${ }^{(3)}$

Currently, the management for delirium is multifactori$\mathrm{al}^{(4)}$ and should include the treatment of reversible conditions and addressing specific patient and care factors which may be contributing to the delirium. ${ }^{(4)} \mathrm{A}$ recognized component of this approach, both for the prevention and in the management of delirium, involves the employment of constant observation (CO) (also known as special observation). ${ }^{(4,5)}$

The concept of CO has been around for some time, ${ }^{(2,6)}$ and associated definitions have ranged from 'an intervention in which continuous one-to-one monitoring is used to assure the safety and well-being of an individual patient or others', ${ }^{(7)}$ to 'direct observation of patients for the purpose of providing a safer environment for the patient'. ${ }^{(8)}$ However, a more practical description for $\mathrm{CO}$ uses $\mathrm{CO}$ for the provision of one-to-one observation by a constant observer (a dedicated person employed to sit with the patient). Once mostly reserved for use within the psychiatric setting for patients at high risk of self-harm or suicide, ${ }^{(9)}$ the role of $\mathrm{CO}$ has diversified and is now most commonly implemented for the management of delirious patients. ${ }^{(4)}$ The term 'sitter' is used to refer to the person providing constant observation. Whilst numerous different terminologies exist for these constant observers (i.e., patient sitters, volunteers, client attendants, patient attendants, companions), for the purpose of this review they will be referred to as sitters.

Therefore, our primary objective for this literature review is to provide an overview of the current role of the sitter within the management of delirium in hospitalized patients by first addressing the current understanding of the role for sitters, the type of person employed in this role, indications 
for current usage, and the frequency they are used. Secondary objectives include the identification of key training requirements required for the sitter role and establishment of the cost-effectiveness of their role within delirium management.

\section{METHODS}

A literature search was performed of MEDLINE, Cochrane Database of Systematic Reviews, and PubMed from the years 1960 to October 2011. The definition for sitter used in the articles was accepted for this review. The following search terms were used: sitter / patient sitter / companion / nurses' aides / special care aides, confusion / delirium / acute confusional state, responsibilities / roles, constant observation / observation, outcomes, intervention, cost / cost analysis. A large number of search terms were used in order to ensure completeness, with identification and inclusion of all potential studies. Inclusion criteria required the articles (and abstracts) to be in the English language and contain relevant research involving at least one of the following: the roles of the sitter, people used for the sitter role, current usage of sitters, indications/assessment and/or tools/evaluation for sitter use, education/training requirements/pre-requisitions required for the role, costs associated with sitter use, sitter use/role within delirium setting including non-pharmalogical intervention models for delirium management. One additional article addressing accuracy of nursing staff for detecting delirium was also included due to the significant impact this could have for sitter usage. One reviewer was responsible for data collection, data analysis, and performing the literature review. Quality assessment was made based on: study design (and blinding), sample size, methodology, and validity of results. From a total of 147 citations initially identified, 50 full-text manuscripts were reviewed and 37 articles were included in the final analysis. A meta-analysis was not conducted due to clinical heterogeneity between included articles. Instead, a summary of the data is presented (Table 1).

\section{RESULTS}

\section{The Sitter Role}

The term 'sitter' relates to the person performing the one to one observation. ${ }^{(8)}$ One of the first reports documenting sitter use was in 1985 as part of a nursing intervention for post-operative orthopedic patients, in which a 'nurse visitor' was employed to provide consistent contact with patient. ${ }^{(10)}$ Whilst their presence may not have significantly influenced the rate or duration of delirium, it does provide evidence supporting the use of patient sitters in the management of delirium. Currently, there is no guidance available regarding who should be used in the sitter role, and no evaluation has been performed to assess the impact that the type of person in this role has upon the patient's clinical outcome; as a result the type of sitter used is determined by the local health region.
The current literature describes a variety of people having been used in the sitter role. The use of skilled nursing staff or nurses' aides as sitters has been well-described. ${ }^{(2,11)}$ Whilst the experience and training they could offer is vast, their use is significantly restricted by an associated high-cost expenditure combined with limited staffing levels. ${ }^{(12)}$ Family members or other relatives are used quite often, inadvertently, as sitters by health-care staff, which is supported by a study that looked at family participation in patient care in the hospital setting and showed their involvement ranging from direct provision of patient care to companionship. ${ }^{(13)}$

One study based in Australia used volunteers in the combined role of observers and companions for patients who had been highlighted as being at a high risk of falling. (14) These volunteers remained with them in the room and were involved in patient interaction, in addition to alerting nursing staff any time patients were identified as being about to fall. Whilst the results from this study showed significant reductions in patient fall rates, limited availability of these volunteers, combined with significant restrictions imposed from occupational health and safety regulations on their role, may limit their use in this capacity.

\section{Current Sitter Usage}

Although once used for observation of high-risk psychiatric patients, the use of sitters is now most commonly employed as part of the management for delirious patients. Another established, albeit less frequently recognized role, is the provision of companionship for patients in palliative care. ${ }^{(14)}$ Alternative uses for the sitter role have been seen as part of a preventative strategy for 'wandering' patients ${ }^{(8)}$ and in fall prevention. ${ }^{(15,16)}$ An alternative role for the sitter has included their use for 'rooming' with patients. This is when a family member or close friend stays in the patient's room, and has been shown to be effective for elderly orthopaedic patients, as described by one study on post-operative elderly orthopaedic patients to assess its clinical impact on delirium rates. (6) Although hospital length of stay and duration of delirium were not significantly reduced, it was shown to be feasible and may possibly be most beneficial for the acutely admitted elderly population. More recently, successful sitter use was shown in the role of a 'rehabilitation patient companion' on an acute brain injury unit in an attempt to reduce costs and improve patient and nurse satisfaction. ${ }^{(17)}$

\section{Sitter Education and Training}

An absence of national guidelines or regulations governing sitter use has inevitably led to a lack of specific criteria (including indications) for hiring sitters, resulting in decisions being made at the local level. This lack of standardized criteria regulating the use of sitters and the absence of formal guidelines (provincial and federally) has highlighted an important deficit in the system that requires addressing. 
TABLE 1.

A summary of studies included in final analysis

\begin{tabular}{|c|c|}
\hline Reference & Study Details \\
\hline $\begin{array}{l}\text { Delirium: an } \\
\text { independent } \\
\text { predictor of } \\
\text { functional decline } \\
\text { after cardiac } \\
\text { surgery }{ }^{(1)}\end{array}$ & 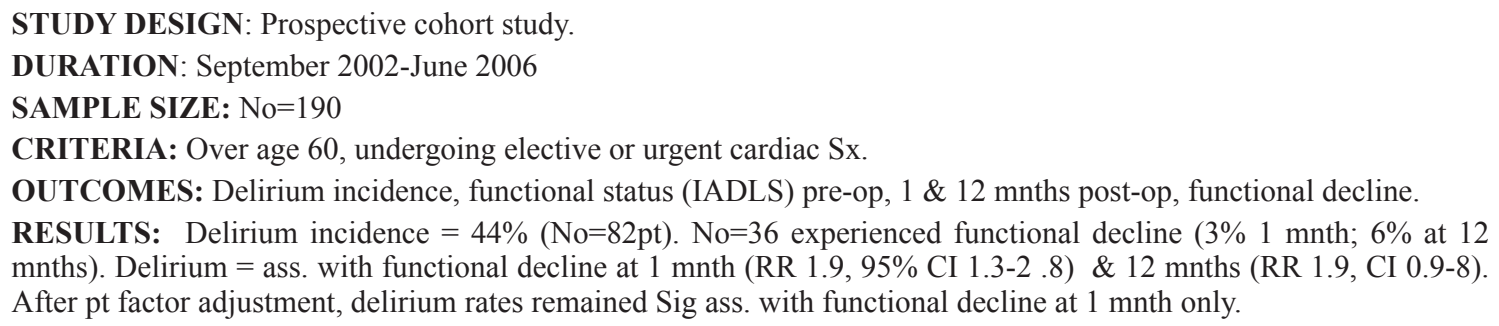 \\
\hline $\begin{array}{l}\text { Constant } \\
\text { observation }(\mathrm{CO}) \text { : } \\
\text { maintain safety, } \\
\text { lower costs }\end{array}$ & $\begin{array}{l}\text { STUDY DESIGN: } 2 \text { Prospective studies performed in single setting. } \\
\text { DURATION: } 5 \text { months }(1995) \text {. } \\
\text { SAMPLE SIZE: } \mathrm{No}=231 \\
\text { CRITERIA: Pts admitted to a teaching hospital, requiring CO. } \\
\text { OUTCOMES: Constant Observer usage (COu); Cost expenditure for Ext. Agency (ExA) \& budgeted Nursing } \\
\text { Assistants (NurA). } \\
\text { SITTERS: ExA or NurAs. Training requirements dictated by local agency. } \\
\text { INTERVENTION: Use by CO staff of a set of } 8 \text { per-specified interventions. } \\
\text { RESULTS: Intervention = reduction in COu by ExA. Total cost expenditures decreased }(\$ 43,445) \text { with an ass. increased } \\
\text { usage of NurA }(\$ 7988) \& \text { total net cost savings }=\$ 35466 \text {. }\end{array}$ \\
\hline
\end{tabular}

The cost of delirium STUDY DESIGN: Prospective cohort study.

in the surgical patient $^{(3)}$

SAMPLE SIZE: $\mathrm{No}=500$

CRITERIA: Over age 50; inpatient elective non-cardiac surgery; predicted LOS greater than 2 days; English speaking.

OUTCOMES: Delirium incidence; LOS; Costs.

INTERVENTION: Pre-op assx then daily screening of pts for delirium from day 1 to day 4 post op.

RESULTS: Delirium incidence $\mathrm{N}=57(11.4 \%)$. Mean LOS was $\operatorname{Sig}(\mathrm{p}<0.001)$ greater for delirious pts $(6$ days vs. 4.6 days), $\&$ overall costs greater in the delirium group. Multiple regression analysis $=\mathrm{N} / \mathrm{S}$ difference in either LOS, total $\&$ direct costs.

A multicomponent intervention to prevent delirium in hospitalized older patients $^{(4)}$
Modified Hospital

Elder Life

Program: effects on abdominal surgery patients $^{(5)}$
STUDY DESIGN: Controlled non-randomized clinical trial.

DURATION: 3 years (March 1995 to March 1998).

SAMPLE SIZE: $\mathrm{No}=852$ (Intervention group (IG) $\mathrm{No}=426$; Control group (CG) $\mathrm{No}=426$ ).

CRITERIA: Over age 70; admitted to general medical service; absence of delirium at presentation.

OUTCOMES: Delirium incidence, delirium duration, No. delirium episodes, delirium severity, delirium recurrence rates, adherence to intervention, cognitive impairment (CI), medication usage.

SITTERS: Fully trained volunteers, receiving regular evaluations.

INTERVENTION: Based on "The Elder Life Program", involved the use of an interdisciplinary team of trained individuals \& implementation of a set of standardized protocols for the management of 6 risk factors for delirium.

RESULTS: Delirium incidence $=9.9 \%$ (IG) vs. $15.0 \%$ (CG). No. of days with delirium (161 vs. 105 respectively)) \& No. delirium episodes (90 vs. 62) was Sig greater in CG vs. IG $(\mathrm{P}<0.02 ; \mathrm{p}<0.03)$. N/S difference $=$ delirium severity or recurrence rates between groups. Adherence to the intervention $=87 \%$. Sig. improvement occurred in the degree of $\mathrm{CI}$ in patients with B/L CI. Sig. reduction = usage of sleep medications for all pts.

STUDY DESIGN: Pre/post intervention clinical trial.

DURATION: 20 months (August 2007-April 2009).

SAMPLE SIZE: $\mathrm{No}=179(\mathrm{IG}=102 ; \mathrm{CG}=77)$.

CRITERIA: Over age 65; admitted to surgical ward for elective abdominal Sx; LOS greater than 6 days.

OUTCOMES: Functional status change, changes in nutritional status \& cognition, body weight, grip strength, delirium rates.

SITTERS: Trained HELP Nurses.

INTERVENTION: Based on modified HELP model \& conducted by HELP-trained nurses. Consisted of: early mobilization, nutritional assistance, and therapeutic (cognitive) activities.

RESULTS: Sig. decrease in functional decline. No change = nutritional status (Sig) independent of the B/L function, education, dx, co-morbidities, procedure or duration of Sx. Delirium rates= Sig. lower in IG group (0\%) vs. CG (16.7\%) $(\mathrm{p}<0.001)$. 
TABLE 1.

Continued

Reference

Rooming-in for elderly surgical patients $^{(6)}$

Constant observation in the general hospital( ${ }^{(7)}$

Observation assistants: sitter effectiveness and industry measure ${ }^{(8)}$

Decreasing the costs of constant observation $^{(9)}$

Reducing acute confusional state in elderly patients with hip fractures ${ }^{(10)}$
STUDY DESIGN: Prospective randomized trial.

DURATION: 10 mnths.

SAMPLE SIZE: $\mathrm{No}=24(\mathrm{IG}=13 ; \mathrm{CG}=11)$.

CRITERIA: Over age 60; admitted to orthopedics; $\mathrm{dx}$ of U/L limb fracture requiring unplanned Sx / planned LE joint replacement; English-speaking family member/close friend willing to stay overnight.

OUTCOMES: Delirium incidence, complication rate, LOS, B/L mental status (MS), functional status, co-morbidity (ChI).

SITTERS: Aka 'Roomers' - close friends / family members.

INTERVENTION: Sitters would 'room' with patient (i.e., stay overnight for 4 or more of the first 7 nights).

RESULTS: N/S differences between the two groups for delirium incidence, complications or LOS. B/L MS was a Sig. covariate. IG pts with unplanned admissions $=$ shorter LOS. Patients with planned surgeries $=$ shorter LOS $\mathrm{w} / \mathrm{o}$ rooming-in intervention. N/S differences between groups for sleep quality, length or frequency of nursing checks for pts.

STUDY DESIGN: Chart review.

DURATION: 9 mnth period (Oct 1 1993-June 1994).

SAMPLE SIZE: $\mathrm{No}=115$.

CRITERIA: Pts requiring CO.

OUTCOMES: $\mathrm{CO}$ indications, duration of $\mathrm{COu}$, pts. behaviour with $\mathrm{CO}$, medication usage, restraint use, $\mathrm{CO}$ cost (hourly rate).

SITTERS: Ext. agency staff \& own staff on overtime.

RESULTS: Most common indication for $\mathrm{CO}=$ organic mental syndrome. Mean duration for $\mathrm{COu}=13.9$ days. Sig. indicators predicting need for $\mathrm{CO}=$ disorientation, psychiatric medication use $\&$ absence of ETOH use. Average cost = $\$ 3,415$ per incident (range $\$ 144-\$ 68,500$ ); median cost $=\$ 1,872$.

DESIGN STUDY: Quality Improvement (QI) study.

DURATION: 8 mnths (Oct 2009-Mar. 2010).

SAMPLE SIZE: $\mathrm{No}=38$

CRITERIA: High risk psychiatric (HRP) pts (SADPERSONS Scale) \& high risk fallers (HRF) pts (MORSE fall risk scale).

OUTCOMES: No. elopements, No. documented assaults, fall rate (No. falls/1000 pt days), sitter usage for HRF \& HRP using the Average daily consensus (ADC) for actual hours worked \& demand hours, sitter costs.

SITTERS: Paid employees from sitter bank. Previous training essential with 30 mins additional training prior to commencing.

INTERVENTION: Sitter usage on safety outcomes for HRP \& HRF patients.

RESULTS: No elopements / assaults documented. Fall rates fluctuated during study period (704 Oct 2009 to 917 falls/ pt days March 2010). No correlation in sitter usage between actual ADC and demand ADC for fallers. No correlation between demand $\mathrm{ADC}$ for $\mathrm{HRF} / \mathrm{HRP}$ and their respective actual ADCs. Sitter costs decreased $=12.4 \%$ post intervention.

STUDY DESIGN: Performance Quality Project.

DURATION: May-Aug 2008.

SAMPLE SIZE: $\mathrm{No}=175$.

CRITERIA: Pts admitted to medical, surgical unit, ICU, rehabilitation \& women care/ obstetrics units.

OUTCOMES: No of CO shifts, No of falls, restraint use.

INTERVENTION: Psychiatric liaison nurse consult for patients requiring $\mathrm{CO}$.

RESULTS: Delirium \& confusion precipitated most CO consults (62\%); suicidal ideation \& elopement risk accounted for remainder. Decreased No. of CO shifts $(1,280$ to 606) \& decreased fall rate. Total cost savings $=\$ 97,056$ over a $4 \mathrm{mnth}$ period incl. $53 \%$ reduction in $\mathrm{CO}$ costs.

STUDY DESIGN: Prospective trial

SAMPLE SIZE: $\mathrm{No}=5$.

CRITERIA: Over age 60; admitted to orthopedic unit across three hospitals.

INTERVENTION: Interpersonal \& environmental nursing interventions.

RESULTS: Incidence of confusion decreased from $51.5 \%(\mathrm{CG})$ to $43.9 \%$ (IG). After controlling risk factors $=$ Sig decreased incidence of confusion $(\mathrm{p}<0.02)$. Most effective interventions $=p t$ re-orientation, correcting sensory deficits $\&$ increasing continuity of care. 
TABLE 1.

Continued

Reference

Constant

observation in the general hospital: a review $^{(11)}$

Registered nurses' job demands in relation to sitter use: nested casecontrol study ${ }^{(12)}$

Using family visitors, sitters, or volunteers to prevent inpatient falls ${ }^{(13)}$

A sitting/ companionship service for palliative care patients $^{(14)}$

A volunteer companion

- observer intervention to reduce falls ${ }^{(15)}$
STUDY DESIGN: Review article.

OUTCOMES: Role of $\mathrm{CO}$ in hospital, indications for $\mathrm{CO}$, responsibilities \& training for $\mathrm{CO}$, whom should perform $\mathrm{CO}$, assx for the need \& usage of $\mathrm{CO}$.

SITTERS: Family members, RGN, security \& volunteers with variable amounts of training.

RESULTS: CO mostly used as a therapeutic intervention with family education. Confusion $=$ most common indication for CO.

Using staff for CO staff is intensive and requires full work based training, thus the need for CO should be reviewed daily.

STUDY DESIGN: Nested case-control study.

DURATION: 23 mnths (Jan 2007-Dec 2008).

SAMPLE SIZE: $\mathrm{No}_{0}=5346$.

CRITERIA: Over age 18; admitted to medical / surgical unit.

OUTCOMES: RN overtime, RN absenteeism, work experience, sitter use.

SITTERS: Ext. agency hired using sitter payment bank.

INTERVENTION: Impact of sitter usage on RN demands.

RESULTS: Pts. with assigned sitter ass. with high rates of RN overtime, absenteeism \& lower RN cumulative experience. Each additional hr. of RN overtime = increased LH of sitter use by $108 \%$ (OR =2.08, 95\% CI: 1.32-3.29). Every 5 yrs. of collective RN experience reduced odds of sitter use $=23 \%(\mathrm{OR}=0.77,95 \% \mathrm{CI}=0.66-0.89)$.

STUDY DESIGN: Cross sectional design survey study.

DURATION: 1 month (May 2006).

SAMPLE SIZE: $\mathrm{No}=101$

CRITERIA: Over age 21; voluntary participation.

OUTCOMES: Family participation, roles \& involved activities of family members.

INTERVENTION: 1 page questionnaire.

RESULTS: Participation $=78 \%$ were female $(\mathrm{No}=78)$. Average age $=42 \mathrm{yr}$. $40.4 \%$ of family participants were pt.'s children. $40.4 \%(\mathrm{No}=38)$ employed family members. $59.6 \%(\mathrm{No}=59)$ took turns keeping pt company. Most common role $=$ provision of physical care $(87.9 \% ; \mathrm{No}=87) \&$ psychological support $(80.8 \%$; No=80). $60.6 \%(\mathrm{No}=60)$ were involved in communication with the medical team.

STUDY DEISGN: Retrospective study.

DURATION: 1992-1993.

SAMPLE SIZE: $\mathrm{No}^{=} 6$

CRITERIA: All new volunteers whom had recently attended in-house training program.

OUTCOMES: Reasons for volunteering, evaluation of program.

SITTERS: Patient-companions consisted of volunteers who had attended required training course.

INTERVENTION: Evaluation of the training program using a questionnaire \& evaluation tool.

RESULTS: Main reason for involvement $=$ previous experience $(\mathrm{No}=5)$, support for community $(\mathrm{No}=1) \&$ personal reasons $(\mathrm{No}=3)$ incl. 'wanting to repay the kindness shown to them \& family', supporting the organization \& wanting to help patients \& carers. Training of volunteers was well received.

STUDY DESIGN: Pilot prospective descriptive study

DURATION: 6 mnths; later extended to 18 months.

SAMPLE SIZE: $\mathrm{No}=26$.

CRITERIA: High risk pts identified by nursing staff.

OUTCOMES: Fall rates/1000 patient days, companion-observer $(\mathrm{COb})$ evaluation \& satisfaction.

SITTERS: $\mathrm{COb}=$ volunteers with no formal training; only pre-requisite was criminal security check.

INTERVENTION: High risk pts requiring $\mathrm{CO}$ were placed in rooms with the $\mathrm{COb}$ whose role was for $\mathrm{CO} \&$ patient interaction.

RESULTS: Intervention $=$ reduction in fall rates by $51 \%$ leading to project extension by 18 mnths. Extended results $=$ Sig reduction in fall rates by $44 \%(\mathrm{p}<0.000)$. No falls occurred in room with $\mathrm{COb}$. COb liked their role. Issues identified by the evaluation incl. a preference to working in pairs $\&$ the need for better definition of their role. 
TABLE 1.

Continued

Reference

Can volunteer companions prevent falls among inpatients? A feasibility study using a pre-post comparative $\operatorname{design}^{(16)}$
A creative alternative for providing constant observation on an acute-brain-injury unit $^{(17)}$

Ensuring the competence of one to one sitters ${ }^{(18)}$
Accuracy of nurse documentation of delirium symptoms in medical charts ${ }^{(19)}$
STUDY DEISGN: Feasibility study.

DURATION: Feb-May 2003.

SAMPLE SIZE: $\mathrm{No}=32$.

CRITIERA: High risk patients admitted to the safety bay unit.

OUTCOMES: Ward fall rate (WFR) (falls / 1000 of occupied bed days) measured at B/L \& during implementation, No. hrs. volunteer time, volunteer satisfaction (VS), family satisfaction (FS), Nursing staff satisfaction (NSS).

SITTERS: Unpaid volunteers. Training used 'Volunteer /companion training program'. Role definition provided. INTERVENTION: Volunteer-companion initiative using trained volunteers to observe high risk fall patients.

RESULTS: The WFR: IRR for falls during implementation vs. B/L = 1.07(95\% CI 0.77-1.49) which was N/S $(\mathrm{P}<0.246) .32$ volunteers donated $2345 \mathrm{hr}$. with a predicted cost $\$ 24.25 \mathrm{AD} / \mathrm{hr} \&$ total cost \$56, 866. Evaluation of VS using Journal entries $(\mathrm{No}=19) \&$ survey $(\mathrm{No}=16)$ was positive. $\mathrm{FS}=$ confusion about role of volunteer; only 8 understood their role. All $(\mathrm{No}=20)$ were positive about volunteers. NSS: $(\mathrm{No}=22)$ agreed with usefulness of volunteer's role; 7 = sitter role took up too much time.

STUDY DESIGN: Performance improvement project.

CRITERIA: Over age 13; admitted to the traumatic brain injury (TBI) unit.

OUTCOMES: FIM (functional independent measure), NSS, salary costs associated with CO (calculated from reviewing unit budget), fall \& restraint rates pre \& post implementation.

SITTERS: Rehabilitation patient companion (RPC). Requirements: 44 hours of training provided prior to starting \& previous experience as CSP/state-tested nursing assistant. Role definition provided.

INTERVENTION: RPC used in sitter role, but provided additional assistance $+/$ - interaction. An additional intervention incl. use of a dayroom as an alternative to $\mathrm{CO}$.

RESULTS: Mean FIM score pre-implementation = 32.2; post implementation $=31.36(\mathrm{~N} / \mathrm{S})$. Pt. exposure to therapeutic activities increased. NSS increased. Using the day room $=1$ less staff on unit \& annual savings $=\$ 25,000$. Additional annual cost savings of $\$ 25000$ observed from additional help provided by RPC (decreased used of evening staff). Restraint rates fell (834.9 to 717.2) post-implementation; fall rates remained unchanged.

STUDY DESIGN: Quality Assurance Study.

CRITERIA: Nursing staff \& sitters in specified facilities.

OUTCOMES: Sitter documentation of pt. behaviour, No. Cases, No. sitter hrs, mean hrs/case, average cost/case, no. episodes of aggressive behavior by pts in ER.

SITTERS: Untrained non-clinical staff.

INTERVENTION: $1 \mathrm{hr}$ sitter education classroom sessions covering 3 topics (policy \& procedure, symptom recognition \& risk assessment) followed by post-test evaluation \& handout.

RESULTS: Intervention $=$ better sitter documentation. No. of cases decreased (46 vs.55) \& was cost effective (decreased costs/case (\$476 vs. \$757)). Sitter hours (1825 vs. 3846) \& mean hours (63 to 40) reduced. No aggressive behaviour was seen.

STUDY DESIGN: Descriptive study

DURATION: March 1996 to January 1999.

SAMPLE SIZE: $\mathrm{No}=226$

CRITERIA: Over 65; patients admitted to one of five medical wards; seen w/in $48 \mathrm{hr}$ of admission.

OUTCOMES: Delirium rates (CAM \& SMPQ), nursing documentation of 6 delirium symptoms, delirium severity (Delirium index), prior CI (IQCDE), cognitive status at admission (MMSE), overall health (ChI), functional status.

RESULTS: Delirium incidence $\mathrm{No}=225$. Average delirium severity $=$ mild (DI 7.8). Documentation of delirium symptoms $=$ poor. $64.2 \%$ had $1 /+$ symptoms documented. Disorientation, agitation and altered LOC most commonly documented symptoms. Documentation of disorientation $=$ sens $26.5 \% \& \operatorname{spf}$ of $100 \%$. Univariate analysis showed pts with higher co-morbidities, greater delirium severity and required use of physical restraints $=$ ass. with better charting of delirium symptoms. 
TABLE 1.

Continued

Reference

Effective assessment of use of sitters by nurses in inpatient care settings ${ }^{(20)}$

The Hospital Elder Life Program: a model of care to prevent cognitive and functional decline in older hospitalized patients $^{(21)}$

Recruitment of volunteers to improve vitality in the elderly: the REVIVE study ${ }^{(22)}$

A S.A.F.E alternative to sitters $^{(23)}$
STUDY DESIGN: Retrospective descriptive study.

DURATION: August 2005-Feb 2007.

CRITERIA: Pts. admitted to 2 medical units.

OUTCOMES: Use of sitters, restraint use, fall rate \& fall injury/1000 pt days, nursing hrs; nursing hrs/pt. day, study unit monthly reports, Quarterly reports of national database of nursing quality indicators, PAAT reports.

SITTERS: Average sitter rate $=\$ 13.91$.

INTERVENTION: Implementation of Patient Attendant Assessment tool (PAAT) for completing sitter fill /requests.

RESULTS: N/S difference was found between the 2 units. Unit 1= PAAT Sig improved fill/request rates for sitters $(\mathrm{p}<0.04) \&$ ass. with Sig reduction in soft limb restraint use $(\mathrm{p}<0.02)$ but Sig increased fall rates $(\mathrm{p}<0.01)$. Nursing hrs $(p<0.01) \&$ total nursing hrs/pt day $(p<0.01)$ Sig increased. For unit 2 , only fill rates were Sig improved $(p<0.01)$ whilst $\mathrm{RN}$ hours $(\mathrm{p}<0.01)$ \& total nursing $\mathrm{hr} / \mathrm{pt}$. day $(\mathrm{p}<0.01)$ Sig. increased. A correlation was seen between higher sitter request rates \& lower restraint use.

STUDY DEISGN: Prospective trial

DURATION: March 1995-August 1999.

SAMPLE SIZE: $\mathrm{No}=1507$.

CRITERIA: Over age 70; admission to specified wards; presence of $1 /+$ pre-defined risk factors: CI, any mobility / ADL deficit, dehydration, visual impairment, hearing impairment or ability to communicate.

OUTCOMES: Adherence to intervention, decline in cognitive status, functional decline (ADLs), pt/family satisfaction, delirium incidence; sleep quality; cost benefit.

SITTERS: Hospital volunteers program. Training: $16 \mathrm{hrs}$ (didactic \& small group training \&16 hrs of 1-on-1 training). INTERVENTION: Targeted interventions for identified risk factors implemented by an interdisciplinary team consisting of Geriatric nurse specialist, Elder Life Specialists, trained volunteers \& geriatricians.

RESULTS: Adherence to intervention $=89 \%$. Cognitive decline decreased (8\% (IG) vs. $26 \%(\mathrm{CG})$ ) along with reduced functional decline $(14 \%$ (IG) vs. 33\% (CG)). Pt \& family satisfaction greater than $90 \%$ with substantial positive feedback. The effectiveness of the program for delirium prevention \& non-pharmacological sleep protocol has been previously demonstrated. Preliminary cost savings: $\$ 1500$ / pt/hospitalization.

STUDY DESIGN: 2 controlled before / after prospective studies.

DURATION: Study $1=5 \mathrm{mnths}$ (2003); Study $2=5 \mathrm{mnths}(2004) \mathrm{g}$

SAMPLE SIZE: $\mathrm{No}=37(\mathrm{IG}=16 ; \mathrm{CG}=21)$

CRITERIA: Over age 70; presence of $1 /+$ risk factors (RF) for delirium; ability to communicate; admitted to the geriatric ward.

OUTCOMES: Study 1: Incidence of delirium, delirium severity. Study 2: Nursing assistant use, financial cost.

SITTERS: Help trained volunteers.

INTERVENTION: Study 1=Volunteer-mediated interventions (daily orientation, therapeutic activities, feeding \& hydration assistance, vision \& hearing protocols). Study 2: Assx of impact of the intervention on nursing assistant use.

RESULTS: Study 1: Sig decrease in incidence of delirium $(p<0.032)$ and delirium severity $(p<0.045)$. Study 2 : Reduction in nursing assistant hrs (316hr /month) with cost savings $=\$ 129,186$ annually.

STIUDY DESIGN: Pilot study.

DURATION: 2 months.

CRITERIA: 2 populations: Group 1: individuals requiring enhanced supervision (e.g. non-compliance / safety risks). Group 2: stable neurologically impaired patients requiring close observation prior to rehabilitation.

OUTCOMES: Sitter use rates, restraint use, cost saving.

SITTERS: Healthcare staff that had undergone SAFE training ( 8 hrs. duration).

INTERVENTION: Development \& implementation of a S.A.F.E. unit for cohorting pts into close proximity to staff members.

RESULTS: Sitter use \& restraint use decreased in Pilot study. Mnthly cost of sitters 1 year prior to S.A.F.E. implementation $=\$ 18,301$ vs. 1 year post-implementation $=\$ 3,223$. 
TABLE 1.

Continued

Reference

Patient and nurse staffing characteristics associated with high sitter use $\operatorname{costs}^{(24)}$

The costeffectiveness of a patient-sitter program in an acute care hospital: a test of the impact of sitters on the incidence of falls and patient satisfaction $^{(25)}$

Replicating the Hospital Elder Life Program in a community hospital and demonstrating effectiveness using quality improvement methodology $y^{(26)}$

Multi-component targeted intervention to prevent delirium in hospitalized older patients: what is the economic value ${ }^{(27)}$
STUDY DESIGN: Prospective study.

DURATION: 2007-2008.

SAMPLE SIZE: $\mathrm{No}=1151$

INCLUSION: Over 18yrs; admitted to medicine / surgery; admitted to the specified units.

OUTCOMES: Pt. health / mental conditions ass. with high risk of disruptive behaviour ( 7 categories), pts. at high risk of fall / injurious falls, nursing characteristics, $\mathrm{RN}$ availability, $\mathrm{RN}$ work experience, $\mathrm{RN}$ education, pt care assistant availability, nursing unit characteristics, sitter costs (classified as high $(>\$ 1000)$ or low $(<\$ 1000)$ ).

SITTERS: Paid unlicensed healthcare professionals contracted by ext. agencies.

INTERVENTIONS: Multivariate logistic regression with GEE to estimate relationships.

RESULTS: Median sitter costs $=\$ 772.35$ vs. $\$ 2397$ among pts with high sitter costs. Multivariate analysis $=$ dementia, delirium \& CI (OR 1.49, 95\% CI 1.01-1.2.22) \& schizophrenia pts (OR 2.42, 95\% CI: 1.08-5.76) ass. with increased LH of high sitter costs. Every additional hr worked by RN/pt/day (OR 0.33 (95\% CI: 0.27-0.39)) \& by pt. care assistants (OR 0.11; CI: 0.08-0.15) reduced LH of high sitter costs.

STUDY DESIGN: Retrospective epidemiologic study.

DURATION: 21 months (July 1998 to March 2000).

CRITERIA: Admissions to specified unit.

OUTCOMES: Rate of falls, pt satisfaction with staff \& quality of care, caregiver's response, No. discharges, No. days in quarter, No. staff hrs / patient day, Nursing unit type, No. patient days, No. active beds, No. sitter shifts.

SITTERS: Average sitter rate: $\$ 20 / \mathrm{hr}$

INTERVENTION: Implementation of a patient-sitter program \& its impact on pt falls.

RESULTS: Fall rate increased (0.0029/sitter shift) with an incremental cost $=\$ 0.67$. Pt dissatisfaction decreased $(0.0010 /$ sitter shift $)$ with cost savings $=\$ 0.41$. Pt. dissatisfaction with staff decreased $(0.0029 /$ sitter shift $)$ saving $\$ 1.72$. Decreased pt. dissatisfaction with overall care (0.008/sitter shift), saving $\$ 2.29$. Total net expense $=\$ 56.62$ (cumulative saving of $\$ 3.76$ / sitter shift vs. sitter cost $\$ 160 /$ shift).

STUDY DESIGN: Feasibility study

DURATION: 3.5 years.

SAMPLE SIZE: $\mathrm{No}=4763$

CRITERIA: Age over 70; admitted to specified nursing unit.

OUTCOMES: Delirium rates, cost of care, LOS, Staff satisfaction (Likerttype scale) at B/L \& 6 months, pt (Likerttype Scale).

SITTERS: Volunteers with 6 mnths of training.

INTERVENTION: Multi-component intervention modeled by HELP with sleep, exercise and fluid protocols.

RESULTS: Reduction in delirium rates (AR 14.45\%; RR 35.3\% $(\mathrm{p}=<0.002)$ ). Total cost savings $=\$ 626,261$ over 6 mnths (101 cases saved; \$2,181/case). LOS decreased (3.6 days). Satisfaction scores were 3.3, 4.3, 2.9 (nurses, nurse aids \& patients respectively).

STUDY DEISGN: Primary prevention trial with cost effectiveness analysis.

DURATION: March 1995-March 1998.

SAMPLE SIZE: $\mathrm{No}=852$

CRITERIA: Over age 70; consecutive admissions to specified units; absence of delirium at baseline but intermediate / high risk of developing delirium.

OUTCOMES: Delirium incidence rates during hospitalization, cost effectiveness, personnel costs, equipment costs, health care utilization costs.

SITTERS: As per HELP model.

INTTERVENTION: Multi-component intervention (IG) based on HELP model vs. model of usual care group (CG).

RESULT: Sig reduction in delirium incidence rate in intermediate risk (IR) $(7 \%$ (IG) vs. $12 \%(C G)(p<0.05))$. There was N/S difference in the high risk group (HR) (19\% (IG) vs. 24\% (CG)). Non-intervention costs were reduced Sig for IR group only. 
TABLE 1.

Continued

A volunteer-based Hospital Elder Life Program to reduce delirium ${ }^{(28)}$

Dissemination of the hospital elder life program: implementation, adaptation, and successes $^{(29)}$
Managing delirium and agitation in elderly hospitalized orthopedic patients: Part 2 Interventions $^{(30)}$

Decreasing companion usage without negatively affecting patient outcomes: a performance improvement project $^{(31)}$

Effect of psychiatric liaison nurse specialist consultation on the care of medicalsurgical patients with sitters ${ }^{(32)}$
STUDY DESIGN: Pilot study of HELP model.

DURATION: During 2007.

SAMPLE SIZE: $\mathrm{No}=1334$.

CRITERIA: Age over 70; admissions for longer than 3 days to 1 of 3 medical or surgical wards.

OUTCOMES: Volunteer participation, nursing staff satisfaction (NSS) with program, pt satisfaction (PS).

SITTERS: Volunteers who had attended a training program (incl. video tapes, shadowing \& reverse shadowing).

INTERVENTIONS: Based on the HELP model.

RESULTS: A sustained increase was seen in No. of volunteer (from 24 to 50 ). NSS increased (from $54 \%\left(\mathrm{No}_{0}=45\right)$ to $91 \%(\mathrm{No}=44))$. PS was $95 \%(\mathrm{No}=130)$.

STUDY DESIGN: Cross sectional survey across performed 13 sites.

DURATION: July 12005 - Dec 2005.

SAMPLE SIZE: 13 sites. No=11,344.

CRITERIA: Established, valid HELP dissemination site contract in place for at least 1 year prior to June 302004 , implementation; continued active enrollment of patients.

OUTCOMES: Description of individual hospital sites, description of HELP sites within the hospitals, enrollment procedures including adaptations, HELP interventions, HELP team members \& volunteers, QA, Hospital Outcomes, Program successes, sources of funding, details about specific adaptations.

SITTERS: Volunteers trained using HELP protocol.

RESULTS: HELP had been implemented at 13 sites. 7 sites = teaching hospitals (6 US \& 1 Canadian); remaining sites were non-teaching community hospitals $(7.7 \%) \&$ rural locations $(23.1 \%)$. Many adaptations were required including: enrollment criteria (15.4\%), screening \& assx tools (61.5\%) \& individual intervention protocols (15.4\% to $30.8 \%)$. All sites conducted regular staff meetings. QA procedures occurred in $46.2 \%-92.3 \%$ sites. Multiple advantages were reported following HELP implementation

STUDY DESIGN: Review article.

CRITERIA: Orthopedic elderly pts.

OUTCOMES: Assx of the roles of pharmacological therapy, interpersonal therapy, constant observation, environmental intervention \& restraint use.

RESULTS: Chemical interventions can have a role. Haldol is $1^{\text {st }}$ line for short term use except in ETOH W/D when benzodiazepines are preferred or in select population groups (i.e., risperidone \& Parkinson's). Addressing external environmental factors more important as cognition declines. Caregivers / pt. interaction can be effective for reducing agitation alongside use of family members for CO. Provision of one-to-one CO by sitters for difficult patients is cost effective, esp. when in collaboration with RN. The use of a sitter tip sheet is encouraged. Lack of evidence for other external environmental interventions. Restraint use should only be as a last resort.

STUDY DESIGN: QA project.

DURATION: 12 months (2000-2001).

CRITERIA: Complex medical / surgical patients admitted for short term skilled nursing care $+/$ - rehab.

OUTCOMES: No. companion shifts, companion expense, restraint use, No. falls, ext. agency hired patient companions.

SITTERS: Sitter costs prior to study $=\$ 10.22$ to $\$ 19.33 /$ hour.

INTERVENTION: Implementation of a new assessment tool for hiring sitters (with prior staff training).

RESULTS: Decreased companion use (from over 350 shifts in Oct 2000 to less than 50 in Oct 2001).Companion expenditure costs decreased by $88 \%$ (approx. $\$ 1.15$ million). 3 episodes of restraint use. No. of falls decreased (9 to 5).

STUDY DESIGN: Randomized experimental study.

DURATION: Jan 1988-March 1988 (2 mnths).

SAMPLE SIZE: Total No= 107: (Suicide group $\mathrm{No}=22(\mathrm{IG}=11, \mathrm{UCG}=11))$; Non-suicidal group $\mathrm{No}=85(\mathrm{IG}=36$; $\mathrm{UCG}=49)$ ).

CRITERIA: Pts assigned a sitter for greater than 1 shift on 2/+ consecutive days; admitted to medical/surgical/ obstetric/ gynecological wards. Pts initially randomized to one of two groups (suicidal or non-suicidal group) followed by further assignment into either the intervention (IG) or control (CG) groups.

OUTCOMES: No. sitter shifts, nursing notes observations, No. sitter incidents, No. pt. incidents, LOS.

SITTERS: External sitter pool.

INTERVENTION: Psychiatric liaison nurse (PLN) consultation incl. follow up for duration of sitter use.

RESULTS: N/S differences were found between groups for both the No. of sitter shifts \& No. nursing note observations. Suicidal pts. had Sig. shorter LOS. 
TABLE 1.

Continued

Reference

Perceptions of training for care attendants employed in the people $^{(33)}$

Delirium: effectiveness of systematic interventions ${ }^{(34)}$

Constant observation practices in the general hospital care of older setting $^{(35)}$

STUDY DESIGN: Qualitative study across 2 sites.

SAMPLE SIZE: $\mathrm{No}=80$ (40 per site)

CRITERIA: Specified healthcare staff (care attendants (CA) \& nursing staff) based in two hospitals.

OUTCOMES: Attitudes to training by CA, perceived links between training \& role ambiguity, Nurses involvement in training.

INTERVENTION: Use of two research instruments (focus group discussion and a questionnaire).

RESULTS: Overall positive attitude observed towards training for CA from both nurses \& CAs themselves. A perceived link was determined between the provision of training \& blurring of role boundaries.

STUDY DESIGN: Systematic review.

DURATION: Jan.1987 to Dec.1997.

SAMPLE SIZE: No $=17(10=$ prevention studies $(4$ RCTs; 6 Non RCT); 7 = Detection \& Rx studies $(2$ RCT; 4 NonRCT \& 1 cohort)). Total No pts $=2142$.

CRITERIA: Prevention trials $=$ RCT \& NonRCT; Detection $\&$ Rx trials $=$ RCT, NonRCT \& cohort studies.

OUTCOMES: Prevention studies: Delirium incidence. Detection \& Rx studies: Delirium incidence, post-operative complications, delirium severity, LOS, cognition, anxiety, depression, functional level \& mortality rates.

INTERVENTIONS: A wide range of interventions were used in both the prevention \& detection / Rx studies.

RESULTS: Prevention trials: A large degree of heterogeneity existed between studies; sample sizes ranged from 20 to 235. ARRs for delirium $=13$ to $19 \%$ (median $13 \%$ ) in all surgical patients \& -3 to $3 \%$ elderly medical patients. ARRs for young \& older surgical patients similar. Detection $\&$ Rx trials: length of follow-up $=5$ to 56 days. Detection and $\mathrm{mx}$ of potential etiologic factors had most benefit on cognitive $\&$ functional status in all delirious surgical pts compared to elderly delirious medical pts.

STUDY DESIGN: National survey across 355 hospitals.

DURATION: March-July 1997.

SAMPLE SIZE: $\mathrm{No}_{0}=102$.

CRITERIA: 1 hospital / 743,000 citizens throughout US \& District of Columbia sampled; >200 bedded hospitals.

OUTCOMES: Overall use, expense, staffing patterns, funding strategies cost-saving interventions.

SITTERS: Hired personnel, family members \& volunteers.

INTERVENTION: 4 cost-saving interventions were utilized (utilization of consolidated bed spaces, relocation of pts near nursing stations, placing at-risk pts in bed enclosure devices \& regular toilet assistance).

RESULTS: Almost all responding hospitals employed CO; following implementation, several hospitals had Sig. decreases in CO expenditures. Largest annual decrease $=\$ 340,000$ when low cost personnel used for CO (hired volunteers \&/ or family members). Education was provided to hospital staff re: costs, appropriate use of CO, recognition $\&$ effective rx for delirium

Helping hands: $\quad$ STUDY DESIGN: Pilot study.

CNAs in elder care $^{(36)}$

OUTCOMES: LOS, No. Nursing Home (N/H) discharges, pt satisfaction, Certified Nursing Assistants (CNA) turnover rate, CNA satisfaction.

INTERVENTION: Implementation of a 'Functional Model of Elder Care' by the CNAs.

RESULTS: Intervention $=$ decreased LOS \& N/H discharges. CNA turnover rate fell (from $175 \%$ in 2000 to $20 \%$ in 2004) \& CNA satisfaction increased.

Evidence based guideline: acute confusion / delirium identification, assessment, treatment and prevention $^{(37)}$

STUDY DESIGN: Summary of evidence practice guidelines.

OUTCOMES: Delirium assx, delirium dx, mx options \& preventative strategies.

RESULTS: Delirium assx should involve identification of pts with predisposing \& precipitating risk factors. Confirmation of the $\mathrm{dx}$ is recommended using a recognized tool (i.e. CAM / NEECHAM). If pharmacological therapy is indicated, options include Haldol or atypical antipsychotics; reserve benzodiazepines for Rx in ETOH w/d. Delirium prevention should incorporate evidence based multi-component interventions; if a single component intervention is chosen, then as a minimum this should include education of all involved in patient care.

Abbreviations: $\mathrm{No}=$ number; $\mathrm{Sx}=$ surgery; Mnths=months; IADLS=instrumental activities of daily living; Ass=associated; $\mathrm{RR}=\mathrm{relative}$ risk; $\mathrm{CI}=\mathrm{confidence}$ interval; Sig=significant; $\mathrm{Rx}=$ treatment; Ext=external; LOS=length of stay; Ass $=$ =assessment; W/o=without; N/S=Non-significant; B/L=baseline; $\mathrm{Dx}=$ diagnosis; $\mathrm{U} / \mathrm{L}=$ upper/lower; $\mathrm{LE}=$ lower extremity; $\mathrm{ChI}=$ Charlsons Index; $\mathrm{Rx}=$ treatment; Incl=included; Mins=minutes; LH=likelihood; OR=Odds ratio; Sens=sensitivity; $\mathrm{Spf}=$ specificity; $\mathrm{RCT}=$ randomized control trials; NonRCT=Non-randomized control trials; $\mathrm{Mx}=$ management; W/d=withdraw. 
One study based in the UK looked at sitters currently employed in the palliative care setting and identified the reasons why sitters got involved and showed (indirectly) that higher sitter turnover was linked with inadequate or insufficient training, which supports the need for adequate training for the role..$^{(14)}$

A quality improvement project was done to assess the effectiveness of a one-hour educational program aimed at sitters (and nurses) which addressed key issues including policy and procedure, risk assessment, and symptom recognition, combined with self-learning. ${ }^{(18)}$ In addition to showing improvement through the comparisons of pre- and post-test results, it was also shown to be cost-effective, which supports the use of similar interventions in the provision of sufficient training for sitters.

\section{Employment of Sitters}

The use of sitters is dependent on the ability of nurses or health-care staff effectively recognizing patients requiring constant observation. However, one study, which addressed the recognition by nursing staff of patients with features of delirium by assessing nursing documentation, showed poor documentation of delirium features. ${ }^{(19)}$ Thus, a failure to recognize such patients will inevitably affect the efficiency surrounding the use of sitters.

To help with identification of patients who would benefit from sitter use, and aid and improve sitter requests, an assessment tool, the 'patient attendant assessment tool (PAAT) ${ }^{(20)}$ was created. Using the PAAT, an improvement was seen in completion of the requisition forms, hiring of sitters, and in actual sitter usage, which was accompanied by reduction in restraint use. ${ }^{(20)}$

\section{The Role of Sitters in Delirium}

The use of sitters as part of the multi-interventional approach for delirium has been well established in the Hospital Elderly Life Program (HELP), ${ }^{(21)}$ initially described by Inouye et al. This model used rigorously trained volunteers as part of an interdisciplinary approach to reduce the incidence and rates of delirium. The success of the program was based, in part, on the use of well-trained volunteers for delirium management.

Additional support for the use of sitters is provided from the results of the REVIVE study, ${ }^{(22)}$ aimed at the prevention and reduction of delirium using a multi-interventional approach through the use of trained volunteers to provide patient interaction and re-orientation. The results revealed a reduction in the incidence and duration of delirium, together with a reduced frequency of falls, thus supporting the importance of this approach. In addition, it was associated with a reduced length of stay in hospital, providing indirectly evidence for the use of sitters for cost-saving purposes.

Another similar concept is the Specialized Adult-Focused Environment (S.A.F.E.) units. ${ }^{(23)}$ This model is based on placing all patients who require constant or very close observation in close proximity to one another, either in a single room or unit in the presence of nursing staff, which provides for continuous or close monitoring for these patients. With the implementation of these SAFE units, the use of sitters was decreased significantly, in addition to a reduction in restraint use. A significant cost saving benefit was seen for the health region.

\section{Cost Benefit}

The specific costs pertaining to sitter use are not clear. One study that specifically focused on sitter costs stated median sitter costs as being $\$ 772$ compared to $\$ 2397$ for high sitter use. ${ }^{(24)}$ The interpretation of this information, however, is difficult due to the terminology used for average and high sitter usage for which there is a paucity of data. Certain factors have been associated with higher rates of sitter usage and thus higher sitter costs, which include understaffing of units with registered nurses and certain patient characteristics. This same study proposed that increasing the number of hours worked by skilled nurses or by patient care assistants may reduce use of sitters and thus lead to lower sitter use and lower sitter costs. ${ }^{(24)}$

The cost-effectiveness of a Patient Sitter program was addressed using outcomes which included the frequency of patient falls and patient satisfaction. ${ }^{(25)}$ The results from this study showed only a marginal benefit was gained for both patient satisfaction and fall rates. In this study, the cost for a typical sitter shift was $\$ 160$, which, when adjusted for the (slight) benefit seen in fall rates and patient satisfaction, revealed a net cost expense of $\$ 156.24$.

The HELP program, aimed at reducing the incidence and duration of delirium in hospitalized elderly patients, reduced hospital costs substantially, ${ }^{(26)}$ and has the potential to be replicated in alternative settings from that in which it was first described, possibly making it the most cost-effective way for employing sitters. ${ }^{(26)}$ However, a Cochrane Review looking at the cost-effectiveness of the implementation of similar programs has suggested that whilst this may reduce both incidence of delirium along with costs for 'intermediate' risk patients, there was no significant benefit seen in 'high' risk patients, either in incidence of delirium or cost saving. (27)

\section{Benefits from Using Sitters}

The potential benefit offered by sitters is tremendous, especially considering the provision of constant observation on a busy acute medical ward. The one-to-one monitoring that can be given is invaluable, and provides a safety net for the nursing staff for high risk patients. This is supported by one study which showed the presence of sitters acted as a significant motivator and improved nurses' confidence in ensuring the provision of adequate patient care. ${ }^{(28)}$ 
The results from the REVIVE study ${ }^{(22)}$ provided evidence of the potential benefit that trained volunteers could offer with patient interaction, whilst providing additional benefits in terms of reduced numbers of nursing assistants required, as well as hospital cost savings.

\section{Disadvantages of Sitter Use}

Whilst the role of sitters for use with certain patient population has previously been established, evidence for the cost-effectiveness of sitter usage for patients with delirium compared to traditional management is conflicting. Although the use of sitters may reduce restraint use, their impact on patient fall rates (a major patient safety outcome measure) is marginal at best, ${ }^{(25)}$ and not clinically significant.

One study assessed the use of sitters for patients deemed at high risk of falling and patients in a psychiatric crisis. ${ }^{(8)}$ By identification of patient volume, current sitter usage, and an assessment of demand, the study authors concluded that the use of constant observation with sitters was an ineffective and costly way of attempting to improve patient care. The study further noted that it was more beneficial to identify high-risk patients on admission (using assessment tools), and to train and employ the hospital's own health-care staff as sitters (using a good quality sitter education program) rather than use an external agency. ${ }^{(8)}$

\section{DISCUSSION}

From this review, it is evident that sitters are being employed in a variety of settings. Although there has been no standardized definition created for a 'sitter', a common underlying theme is apparent regarding their role as an observer or companion for the patient - thus providing a potential definition for the sitter role as a person hired 'to provide constant observation or companionship'.

The question of which type of person would provide the most benefit in the sitter role is still not clear. Studies have reported using a variety of people ranging from $\mathrm{RN}$ nurses and special care aides to (untrained) volunteers and patient relatives and family members. However, due to growing demands exerted on nursing duties, there has been an increasing trend towards using external 'hired agency staff' as sitters, with a resulting rise in costs. ${ }^{(2,7)}$

Family members have the potential to be effective sitters; their familiarity with the patient, close relationship, and motivation to stay with the patient can provide essential patient interaction important for delirious patients. In addition, a family member's presence at the bedside can provide close observation, as well as familiarity, and thus enhance patient safety. ${ }^{(13)}$ Their relationship with the patient, however, has the potential to negatively impact patient care if their goals are not the same as the health-care staff.

Volunteers may have the most potential in the capacity of the sitter, provided that they are adequately trained for the role and are freely available, which is evident from success of established program such as HELP model. This is supported by several studies that have justified the use of trained volunteers by showing them to be cost-effective. ${ }^{(22,26)}$ Indeed, the use of sitters relieves the workload of overworked, stressed nursing staff who are often more than happy to have an additional pair of hands in the form of a volunteer sitter. ${ }^{(12)}$

As discussed earlier, sitters are currently employed across the country in several different settings with one of the most common being for delirious patients. Precise details relating to the frequency of the use of sitters at any individual locations are difficult to establish due to a lack of documentation at the local, provincial, and federal levels. This highlights a potential role for the development of a national reporting scheme for recording their use. The absence of a standardized job description for the sitter role and lack of regulations regarding their use have led to the development of a certain degree of unease concerning their use, and accounts for the significant amount of variation and inconsistencies seen in the use of sitters. Without a specific job description for the role of a sitter, health-care institutions are significantly restricted in this capacity, and hindered in benefiting from the use of sitters (i.e., provision of daily social interaction, cognitive stimulation, frequent reorientation therapy, etc.). This is important due to the significant clinical impact that can result following the implementation such interventions would have on delirium outcomes. However, without further clarification (in the form of national guidance) about their position, then these potential benefits offered by sitters cannot be utilized.

Currently there are no formal criteria regulating sitter usage. Sitters are mostly employed as part of the management of delirious patients. Use of sitters for the provision of constant observation for high-risk psychiatric patients is well established. ${ }^{(9)}$ More creative and alternative roles for sitters have been successful, including their use as a 'patient companion' in the palliative care setting ${ }^{(14)}$ and for patients with traumatic brain injury. ${ }^{(17)}$ Although additional roles have used sitters in an attempt to reduce rates of wandering for high-risk patients and patient fall rates, these are theoretical advantages which are not supported by the current evidence available, and conflicts with the general consensus that sitters do not improve frequency of falling and may even increase fall rates; however, the reasons for this are not clear.

The process for identifying those patients who would benefit from the use of sitters has not been well established. The provision of pre-specified criteria or assessment tools to aid nursing staff in recognizing patients who would benefit from these costly resources is important for both reducing costs associated with using sitters and reducing rates of pharmacological therapy with its associated side effects. The importance of appropriate sitter usage is supported by evidence showing that whilst higher sitter usage was associated with decreased rates of soft restraint use, it was accompanied by an alarming increased rate of falls. ${ }^{(20)}$ 
If nursing staff are efficient in recognizing the presence of delirium, then this should naturally improve sitter use and cost-effectiveness. However, whether this happens is debatable. One prospective study looking at the accuracy of nursing documentation relating to their recognition of the signs and symptoms of delirium suggested possible poor recognition, with a possible exception being for severely ill patients. ${ }^{(10)}$ Thus, the poor recognition of delirious patients will affect sitter usage within the hospital setting. Although there are no currently no formal established assessments tools available to aid with this process, there is great potential in the form of a specified assessment tool - the PAAT - as discussed previously, ${ }^{(20)}$ which was created to help assist and thus improve the hiring and requisition of the use of sitters for high-risk patients.

Adequate training for sitters is crucial for clinical, ethical, and financial reasons. Common sense states that the use of untrained sitters in potentially risky situations may have negative consequences. A lack of orientation to either the patient and/or to their role is distracting and problematic for both the patient and sitter and this, combined with the lack of familiarity between them, could create a potentially disastrous situation, as well as being an inefficient use of resources and a hindrance to patient care. In addition, inadequate training for the management of aggressive or agitated patients could put sitters, the patient, and staff at danger and has legal consequences. This is important, as many situations in which sitters are employed (e.g., delirious patients and palliative care) are highly sensitive and emotionally charged settings in which the presence of a sitter can have a big impact on both the patient and relative. This further highlights the extreme importance of sitters receiving adequate training for this role.

As a consequence of the lack of formal/recognized training or assessment, the competence of these sitters has been questioned several times, especially since it is often untrained volunteers that are commonly used in this capacity. ${ }^{(18)}$ The importance of this is evident from the dire consequences that can ensue due to the nature of settings in which these sitters are commonly employed. However, with adequate training, the effectiveness of fully trained volunteers in the sitter role is non-debatable, reconfirming the need for sufficient training for the position.

The employment of sitters in the management of delirious patients may benefit the patient both in terms of delirium control (through the application of consistent, frequent re-orientation therapy), as well as (possibly) reducing or preventing the use of pharmacological agents and/ or restrictive devices. The HELP program has been shown to be successful in both reducing the rate and length of delirium episodes whilst reducing negative clinical outcomes associated with delirium. ${ }^{(21)}$ Similar, smaller programs based on this concept have been set up, ${ }^{(29)}$ the findings of which have shown similar results. Thus, the use of a wellestablished delirium program model for employing trained sitters (i.e., volunteers) may offer the most cost-effective method. However, the translation of a large established program to implementation of smaller similar programs at the local level can be one of the hardest barriers to cross due to restrictions exerted in terms of costs, resistant attitudes from staff, and insufficient staffing levels (to name a few). One article addressed this, ${ }^{(29)}$ and looked at the degree of implementation of similar HELP programs across the country and their success rates. This study showed that only $25 \%$ of those who initially expressed interest in developing the program actually succeeded in establishing an active, functioning HELP program in their local institution.

The variation in sitter usage across the country is considerable. Certainly, in our local region, the use of sitters has been reduced substantially in the last couple of years due to financial restraints. This is hardly surprising considering the severe deficiency of good quality evidence for their use and lack of information supporting their role or even an accurate definition of their position. One study did produce a sitter guidance sheet, stating hints and tips which could offer useful information for current sitters. ${ }^{(30)}$ The impact of this on sitter activity and possible future training would be very interesting to assess. The same review suggested that management of patients who require sitters should avoid placing delirious patients in one single area due to a possible negative influence that may be exerted on one another. However, other studies do not support this concept, which conflicts with evidence from the aforementioned SAFE study. ${ }^{(23)}$

\section{CONCLUSION}

From the 37 articles included in the review, nine studies directly addressed the role for sitters in delirium, with sample sizes ranging from 24-4763. Addressed outcomes included markers of delirium (incidence, severity, duration, number of episodes, and recurrence), patient outcomes (cognitive change, functional change, sleep quality), nursing assistant use, and financial cost. The use of mostly trained volunteers in the sitter role provided the main limitations to these studies. One article addressed training for sitter role in delirium (sample size: 1507) and six articles (sample size: 37-5346) addressed cost-effectiveness for role of sitters in delirium.

Current evidence supports a role for the sitter as part of the management of patients with delirium. Since the majority (and strongest evidence) is seen with the use of trained volunteers in the sitter role as part of an established delirium model (such as HELP), then this may likely be the most costeffective way for advocating their use, possibly in conjunction with use a recognized sitter assessment tool such as the PAAT. The specific type of training required for the sitter role is not clear and requires further research. Certainly, when considering using a sitter, the health-care provider should carefully document the indications for sitter use, and monitor and reassess these indications alongside the frequency and use of the sitter in order to maximize cost-effectiveness. Long 
term, the creation of a national set of regulations or guidelines would provide safeguards in the industry to ensure safe and effective patient care, in addition to the creation of a national reporting scheme for sitter usage.

\section{CONFLICT OF INTEREST DISCLOSURES}

The author declares that no conflicts of interest exist.

\section{REFERENCES}

1. Rudolph JL, Inouye SK, Jones RN, et al. Delirium: an independent predictor of functional decline after cardiac surgery. J Am Geriatr Soc. 2010;58(4):643-49.

2. Turjanica MA, Ardabell TR, Mancini B, et al. Constant observation: maintain safety, lower costs. Nurs Manag. 1998;29(10):82-83.

3. Franco K, Litaker D, Locala J, et al. The cost of delirium in the surgical patient. Psychosomatics. 2001;42(1):68-73.

4. Inouye S, Bogardus ST, Charpentier PA, et al. A multicomponent intervention to prevent delirium in hospitalized older patients. N Engl JMed. 1999;340(9):669-76.

5. Chen $\mathrm{CH}$, Lin MT, Tien YW, et al. Modified Hospital Elder Life Program: effects on abdominal surgery patients. $J \mathrm{Am}$ Coll Surg. 2011;213(2):245-52.

6. Wells N, Baggs JG. Rooming-in for elderly surgical patients. Appl Nurs Res. 1997;10(2):72-79.

7. Blumenfield M, Milazzo J, Orlowski B. Constant observation in the general hospital. Psychosomatics. 2000;41(4):289-93.

8. Harding AD. Observation assistants: sitter effectiveness and industry measures. Nurs Econ. 2010;28(5):330-36.

9. Rausch DL, Bjorklund P. Decreasing the costs of constant observation. J Nurs Adm. 2010;40(2):75-81.

10. Williams MA, Campbell EB, Raynor WJ, et al. Reducing acute confusional state in elderly patients with hip fractures. Res Nurs Health. 1985;8(4):329-37.

11. Jaworowski S, Raveh D, Lobel E, et al. Constant observation in the general hospital: a review. Isr J Psychiatry Relat Sci. 2008;45(4)278-84.

12. Rochefort CM, Ward L, Ritchie JA, et al. Registered nurses' job demands in relation to sitter use: nested case-control study. Nurs Res. 2011;60(4):221-30.

13. Tzeng HM, Yin CY. Using family visitors, sitters, or volunteers to prevent inpatient falls. J Nurs Adm. 2007;37(7-8):329-34.

14. Franks AL, Geary M, Smith I. A sitting/companionship service for palliative care patients: Leicestershire, England. Am J Hosp Palliat Care. 1997;14(4):162-65.

15. Donoghue J, Graham J, Mitten-Lewis S, et al. A volunteer companion-observer intervention reduces falls on an acute aged care ward. Int J Health Care Qual Assur Inc Leadersh Health Serv. 2005;18(1):24-31.

16. Giles LC, Bolch D, Rouvray R, et al. Can volunteer companions prevent falls among inpatients? A feasibility study using a pre-post comparative design. BMC Geriatr. 2006;6:11.

17. Bailey M, Amato S, Mouhlas C. A creative alternative for providing constant observation on an acute-brain-injury unit. Rehabil Nurs. 2009;34(1):11-16.
18. Ragaisis KM, Wedler C. Ensuring the competence of one-toone sitters. J Nurs Care Qual. 1997;11(3):2-3.

19. Voyer P, Cole MG, McCusker J, et al. Accuracy of nurse documentation of delirium symptoms in medical charts. Int J Nurs Pract. 2008;14(2):165-77.

20. Tzeng HM, Yin CY, Grunawalt J. Effective assessment of use of sitters by nurses in inpatient care settings. $J$ Adv Nurs. 2008;64(2):176-83.

21. Inouye SK, Bogardus ST, Baker DI, et al. The Hospital Elder Life Program: a model of care to prevent cognitive and functional decline in older hospitalized patients. J Am Geriatr Soc. 2000;48(12):1697-706.

22. Caplan GA, Harper EL. Recruitment of volunteers to improve vitality in the elderly: the REVIVE study. Intern Med J. 2007;37(2):95-100.

23. Nadler-Moodie M, Burnell L, Fries J, et al. A S.A.F.E. alternative to sitters. Nurs Manage. 2009;40(8):43-50.

24. Rochefort CM, Ward L, Ritchie JA, et al. Patient and nurse staffing characteristics associated with high sitter use costs. $J$ Adv Nurs. 2012;68(8):1758-67.

25. Boswell DJ, Ramsey J, Smith MA, et al. The cost-effectiveness of a patient-sitter program in an acute care hospital: a test of the impact of sitters on the incidence of falls and patient satisfaction. Qual Manag Health Care. 2001;10(1):10-16.

26. Rubin FH, Williams JT, Lescisin DA, et al. Replicating the Hospital Elder Life Program in a community hospital and demonstrating effectiveness using quality improvement methodology. J Am Geriatr Soc. 2006;54(6):969-74.

27. Rizzo JA, Bogardus ST, Leo-Summers L, et al. Multicomponent targeted intervention to prevent delirium in hospitalized older patients: what is the economic value. Med Care. 2001;39(7):740-52.

28. Sandhaus S, Zalon ML, Valenti D, et al. A volunteer-based Hospital Elder Life Program to reduce delirium. Health Care Manag (Frederick). 2010;29(2):150-56.

29. Inouye SK, Baker DI, Fugal P, et al. Dissemination of the hospital elder life program: implementation, adaptation, and successes. J Am Geriatr Soc. 2006;54(10):1492-99.

30. Segatore M, Adams D. Managing delirium and agitation in elderly hospitalized orthopaedic patients: Part 2-Interventions. Orthop Nurs. 2001;20(2):61-73.

31. Salamon L, Lennon M. Decreasing companion usage without negatively affecting patient outcomes: a performance improvement project. Medsurg Nurs. 2003;12(4):230-36; quiz 237.

32. Talley S, Davis DS, Goicoechea N, et al. Effect of psychiatric liaison nurse specialist consultation on the care of medical-surgical patients with sitters. Arch Psychiatr Nurs. 1990;4(2):114-23.

33. Coffey A. Perceptions of training for care attendants employed in the care of older people. J Nurs Manag. 2004;12(5):322-28.

34. Cole MG. Delirium: effectiveness of systematic interventions. Dement Geriatr Cogn Disord. 1999;10(5):406-11.

35. Worley LLM, Kunkel EJS, Gitlin DF, et al. Constant observation practices in the general hospital setting. A national survey. Psychosomatics. 2000;41(4):301-10. 
36. Weitzel T, Robinson SB, Henderson L, et al. Helping hands: CNAs in elder care. Nurs Manage. 2005;36(4):41-44.

37. Sendelbach S, Guthrie PF. Acute confusion/delirium. Identification, assessment, treatment and prevention. J Gerontol Nurs. 2009;35(11):11-18.
Correspondence to: Frances M. Carr, MBChB, Internal Medicine Department, University of Saskatchewan, 704-640 Main Street, Saskatoon, SK S7H 0J7, Canada

E-mail: fmc776@mail.usask.ca 